\title{
ECG-triggered non-enhanced MR angiography of peripheral arteries in comparison to DSA in patients with peripheral artery occlusive disease
}

\author{
Sasan Partovi • Matthias Rasmus • Anja-Carina Schulte • \\ Fabian Rengier - Augustinus Ludwig Jacob - Markus Aschwanden • \\ Christof Karmonik · Geog Bongartz $\cdot$ Deniz Bilecen
}

Received: 3 January 2012/Revised: 10 October 2012/Accepted: 11 October 2012/Published online: 2 November 2012

(C) ESMRMB 2012

\begin{abstract}
Object The purpose of this study was to evaluate peripheral non-enhanced-MRA (NE-MRA) acquired with a 3D Turbo Spin Echo sequence with electrocardiographt (ECG) triggering in comparison to Digital Subtraction Angiography (DSA) as the gold standard in symptomatic peripheral artery occlusive disease (PAOD) patients.

Materials and methods This IRB approved prospective study included 23 PAOD patients from whom three patients had to be excluded. The remaining 20 subjects were included in the analysis (15 male; mean age $62.4 \pm 15.3$ years). The patients first underwent DSA followed by NE-MRA on a 1.5-T whole body scanner within $24 \mathrm{~h}$ after the DSA study. A NATIVE (Non-contrast Angiography of the Arteries and Veins) SPACE (Sampling
\end{abstract}

S. Partovi · M. Rasmus $(\square) \cdot$ G. Bongartz

Institute of Radiology, University Hospital Basel,

Petersgraben 4, 4031 Basel, Switzerland

e-mail: mrasmus@uhbs.ch

\section{S. Partovi}

e-mail: sasanp@gmx.de

G. Bongartz

e-mail: bongartzg@uhbs.ch

\section{A.-C. Schulte $\cdot$ D. Bilecen}

Institute of Radiology, Kantonsspital Bruderholz, 4101 Bruderholz, Switzerland

e-mail: acschulte@web.de

D. Bilecen

e-mail: deniz.bilecen@unibas.ch

\section{F. Rengier}

Department of Diagnostic and Interventional Radiology, University Hospital Heidelberg, Im Neuenheimer Feld 110, 69120 Heidelberg, Germany

e-mail: fabian.rengier@web.de
Perfection with Application Optimized Contrast by using different flip angle Evolution) sequence at four levels (pelvis, upper leg, knee region and lower leg) was acquired. For evaluation purposes, subtracted standardized MIP (maximum intensity projection) images were generated from the NE-MRA data sets. Qualitative assessment of NE-MRA images in reference to the corresponding DSA images, as well as blinded stenosis grading of preselected segments in NE-MRA images were performed by two experienced readers. Image quality in 95 corresponding arterial segments was rated from 1 (good) to 4 (inadequate) directly comparing the NE-MRA with the corresponding DSA segment as the gold standard. Blinded stenosis grading consisted of 66 preselected stenoses rated from 1 $(<10 \%)$ to $4(>90 \%)$ in NE-MRA which were compared to the grade in the corresponding DSA.

\section{A. L. Jacob}

Swissintervention Zentrum für Mikrotherapie, Klinik Hirslanden, Witellikerstr. 40, 8032 Zurich, Switzerland

e-mail: jacob@swissintervention.ch

M. Aschwanden

Institute of Angiology, University Hospital Basel,

Petersgraben 4, 4031 Basel, Switzerland

e-mail: MAschwanden@uhbs.ch

C. Karmonik

Department of Neurosurgery, The Methodist Hospital, 6560 Fannin ST944, Houston, TX 77030, USA

e-mail: CKarmonik@tmhs.org 
Results The mean image quality of NE-MRA in comparison to DSA was $2.7 \pm 1.1$ (reader 1) and $3.0 \pm 1.0$ (reader 2). The kappa value indicating interobserver agreement was 0.34 ; readers 1 and 2 rated the image quality as good in $21 \%$ and $3 \%$, sufficient in $19 \%$ and $41 \%$, limited in $29 \%$ and $14 \%$ and inadequate in $31 \%$ and $42 \%$, respectively. Stenosis graduation revealed significantly higher grades in NE-MRA (reader 1: $3.0 \pm 0.7$, $p<0.001$ and reader 2: $3.1+0.8, p<0.001)$ compared to DSA (mean value DSA $2.7 \pm 0.8$ ). The kappa value indicating interobserver agreement concerning stenosis grading was 0.59 .

Conclusion NE-MRA revealed a relatively high number of inadequate quality segments. This is in line with recently published comparable studies of the similar SPACE NE-MRA techniques. Further advance of NE-MRA techniques remains desirable for patients with PAOD.

Keywords Magnetic resonance angiography (MRA) . Non-enhanced MRA · Peripheral MRA · NATIVE SPACE - Peripheral artery occlusive disease (PAOD)

\section{Introduction}

Contrast-enhanced magnetic resonance angiography (CE-MRA) dominates most MR vascular imaging techniques due to its wide ranging application possibilities [1-5]. Non-enhanced techniques, such as TOF (time of flight) or phase-contrast MRA, played only a minor clinical role in the viewing of peripheral artery imaging over the last decade. Their application for body imaging is limited by motion artifacts due to the longer acquisition times required [6], as well as larger fields of view like the peripheral arterial system.

In spite of the successful integration of CE-MRA into daily routine, there are reasons to reevaluate non-enhanced MRA techniques for peripheral MRA. First, the appearance of nephrogenic systemic fibrosis (NSF) [4, 7-11] in some patients with chronic kidney disease (CKD) following administration of Gadolinium-based contrast agents (Gd-CA) led to a reassessment of the risk profile of Gd-CA use in CKD patients. The coincidence of peripheral artery occlusive disease (PAOD) and CKD is high. Thus nonenhanced techniques are desirable as an additional approach, especially for patients with PAOD and CKD. Second, the potential cost reduction due to CA savings should be considered. Third, MRI-guided vascular interventions in the future [12] with repeated arterial visualizations would result in relatively high $\mathrm{CA}$ doses if no accompanying NE-MRA technique is available (e.g. roadmapping).
In this NE-MRA study, we used a SPACE (Sampling Perfection with Application Optimized Contrast by using different flip angle Evolution) sequence which is a modified 3D Turbo Spin Echo sequence (TSE) with variable flip angle refocusing pulses [13, 14]. The SPACE sequences are designed to lower the specific absorption rate (SAR) while maintaining good T2 contrast in long echo trains utilizing variable flip angles. The scope of this study is the evaluation of the SPACE NE-MRA technique using a modified 3D TSE with ECG triggering versus DSA as a gold standard in PAOD patients.

\section{Materials and methods}

Patients

The study was approved by our local ethics committee. After full explanation of the study, written informed consent was obtained from all patients.

Twenty three patients were prospectively enrolled in this study. All patients suffered from PAOD and had a clinical indication for DSA-guided intravascular intervention/percutaneous transluminal angioplasty (PTA). Exclusion criteria included all common MRI contraindications such as metal implants, pace makers, claustrophobia, mental incapacity, pregnancy, etc.

Three patients had to be excluded after DSA and MR imaging due to (1) insufficient DSA data, (2) major motion artifacts in MRA, and (3) technical failure of ECG triggering. The remaining 20 subjects, five women and 15 men, had a mean age of $62.4 \pm 15.3$ years (30-93 years). Of these 20 patients, 18 suffered from intermittent claudications (Fontaine stage IIa: 12 and Fontaine stage IIb: 6). Two of the patients had no anamnestic claudications, but had a bypass stenosis.

In four of the 20 patients, no ankle-brachial index was measurable due to incompressible arteries. In the remaining 16 patients, the average ankle-brachial index on the side of intervention was $0.76 \pm 0.15$. Ankle-brachial index values ranged from 0.5 to 1.05 , with an ankle-brachial index of 1.05 measured in a patient with proximal/pelvic artery stenosis.

Basic clinical data of the 20 investigated patients are listed in Table 1.

\section{DSA}

A modern angiographic unit (Axiom Artis dTA, Siemens Medical Solutions, Erlangen, Germany) was used for DSA imaging. All PTAs were performed by an experienced interventional radiologist and were clinically indicated. 
Table 1 Summary of clinical basis data of 20 patients, shown as percentage of total group

\begin{tabular}{lr}
\hline & $n(\%)$ \\
\hline Risk factors & \\
Smoking history & $13(65)$ \\
Respiratory disease & $3(15)$ \\
Arterial hypertension & $13(65)$ \\
Hyperlipidemia & $12(60)$ \\
Diabetes mellitus & $5(25)$ \\
Severe chronic kidney disease & $1(5)$ \\
Medication & \\
Blood-pressure drug & $14(70)$ \\
Beta Blocker & $8(40)$ \\
Lipid-lowering agent & $11(55)$ \\
Oral diabetic medication & $4(20)$ \\
Insulin & $2(10)$ \\
Ceiling diuretics & $5(25)$ \\
Other diuretics & $6(30)$ \\
Heparin & $5(25)$ \\
Aspirin & $14(70)$ \\
\hline
\end{tabular}

A mean total of $100 \mathrm{ml}$ (individual range $70-150 \mathrm{ml}$ ) of iodinated contrast material (Ultravist 300; Schering, Berlin, Germany) was injected by a power injector/manually.

Only clinically necessary contrast media administration and imaging was performed during the interventions. Thus, the amount of DSA image data was limited compared to that of NE-MRA image data. Of ten potential vessel segments per patient (five on each side, see image analysis), between two and ten were depicted in each of the DSA images. The average number of visualized segments per patient was 4.75 , resulting in a total of 95 segments.

\section{MRI}

MR imaging was performed on a 1.5-T whole body scanner (Magnetom Espree, Siemens Medical Solutions, Erlangen, Germany) within $24 \mathrm{~h}$ after DSA. An 8-channel CP matrix coil (i.e. four levels with two CP elements each) in combination with a flexible CP coil (4-element design) was used.

The NATIVE (Non-contrast Angiography of the Arteries and Veins) SPACE sequence (Siemens Medical Solutions, Erlangen, Germany) is a modified 3D Turbo Spin Echo sequence.

This variable flip angle fast spin-echo sequence (FSE) has recently been used for the ECG-gated spin-echo-based MR angiography technique $[15,16]$. Although the original ECG-gated FSE-based NE-MRA use the constant refocusing flip angle at 1.5T [17], application of the variable flip angle technique in NE-MRA can be used to reduce the specific absorption rate (SAR) [18]. In addition, it has the great advantage of shortening echo spacing, mainly due to nonselective rectangular refocusing pulses, resulting in reduction of readout duration $[15,16,19]$. It subsequently decreases vessel blurring and performs more accurate data sampling $[15,16]$ as compared to half-Fourier acquisition single-shot turbo spin-echo (HASTE) sequences [20].

This NE-MRA imaging sequence is based on the subtraction of ECG-triggered image data with and without arterial peak flow $[15,16]$. Two NATIVE-SPACE datasets with different ECG trigger delay (TD) are acquired: one with high arterial signal during diastole and one with arterial flow void during systole. By subtracting both datasets, background and venous signal can be eliminated, resulting in an image with bright arterial vessel visualization (Figs. 1, 2, 3).

Prior to NE-MRA imaging, a so-called NATIVE TD CINE scout at pelvis and at knee level was acquired [21]. This is a retrospectively gated cine flash sequence using a large flip angle. It consists of a series of axial single-slice images in various cardiac phases to determine the correct TD time between systolic and diastolic flow by repeated acquisition with increasing TD covering one RR cycle. Optimal TD is estimated by evaluation of the lowest and highest intra-arterial signal during the RR cycle.

Transverse NATIVE SPACE slabs were acquired at four levels always in the same order: Pelvis, upper leg, knee region, lower leg. The following acquisition parameters were used at pelvis level: repetition time $300 \mathrm{~ms}$; echo time $38 \mathrm{~ms}$; slice thickness: $1.5 \mathrm{~mm}$; slices per slab: 64; field-ofview: $400 \times 380 \mathrm{~mm}^{2}$; bandwidth: $868 \mathrm{~Hz} /$ pixel; echo spacing: $2.76 \mathrm{~ms}$; voxel size: $1.6 \times 1.3 \times 1.5 \mathrm{~mm}^{3}$; parallel imaging with GRAPPA acceleration factor of 2 ; variable flip angle. Similar imaging parameters were used for the other steps. An overlap between adjacent steps of 15-20 cm depending on the individual body length was chosen. Imaging parameters of all four levels are listed in Table 2.

For each level, the NATIVE SPACE sequence acquires one data set, which includes two different TDs (systolic and diastolic triggered images). The acquisition time of one step varied individually depending on the heart rate and the imaged level. It was approximated $2-3 \mathrm{~min} / \mathrm{step}$. The total acquisition duration (scout images, $2 \times$ CINE scouts, 2 TDs $\times 4$ steps $\times$ NATIVE SPACE) was 30-40 min.

After data acquisition, inline subtraction and subsequent standardized maximum intensity projection (MIP) reconstruction were performed using the manufacturer's software. Thirteen MIPs were reconstructed at angles of 15 degrees.

Image analysis

Images were independently and separately assessed by two radiologists (both with more than 10 years experience with 
Fig. 1 Schematic overview of ECG-triggered NE-MRA algorithm with vein $(V)$ and artery $(A)$ in dependence from ECG triggering with resulting subtraction of systolic and diastolic image. Upper line: Example source image of systolic flow imaging. Middle row shows amplifications of the right proximal popliteal artery from corresponding diastolic-, systolic-source images and subtraction. Besides MIP reconstruction of right calf arteries of a 58-year-old man with Fontaine stage $2 \mathrm{a}$ prior to PTA

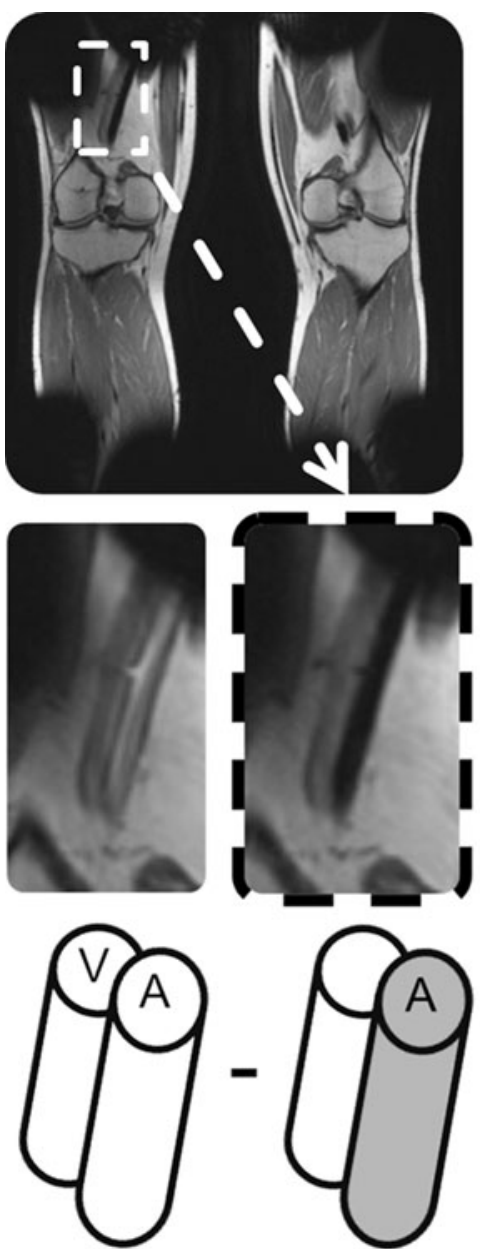

MRA). The previously treated segments with PTA were excluded from the assessment. Image evaluation included two parts: (a) Qualitative assessment of the NE-MRA image in reference to the corresponding DSA image and (b) blinded stenosis grading of preselected segments in NE-MRA images. To reduce bias, stenosis grading was performed at least 1 week before quality assessment. Five arterial segments were defined for image reading: $\mathrm{I}=$ common and external iliac artery, $\mathrm{II}=$ proximal superficial femoral artery, III = distal superficial femoral artery, IV $=$ popliteal artery and $\mathrm{V}=$ calf arteries.

- Qualitative assessment:

The reviewers were asked to give quality grades for each of the 95 comparable segments (i.e. each available DSA step with corresponding NE-MRA images) in direct comparison between NE-MRA and corresponding DSA. Images were presented as subtracted standardized MIP images with corresponding DSA images from one prepared slice and-if desired - all available MRA- and DSA-data. It was decided that MIP reconstructions should be used for the reading sessions to ensure a better comparison with the corresponding DSA image. The reason for this was that those MIP reconstructions are the most similar way in terms of visualization when comparing the gold standard DSA to NE-MRA. The image quality of each segment was evaluated on the basis of a four-point scale with regards to arterial enhancement and the presence of artifacts. The grades do not represent absolute values for NE-MRA image quality, but relative grades of NE-MRA image quality compared to DSA image:

- Grade 1 indicates a good visualization of anatomy and pathology in comparison to DSA.

- Grade 2 indicates a sufficient corresponding visualization of anatomy and pathology with minor limitations due to signal inhomogeneities and/or artifacts in comparison to DSA.

- Grade 3 indicates a limited visualization of anatomy with an insufficient visualization of pathology due to signal inhomogeneities and/or artifacts in comparison to DSA.

- Grade 4 indicates an insufficient correspondence of anatomy and pathology in NE-MRA and DSA. 


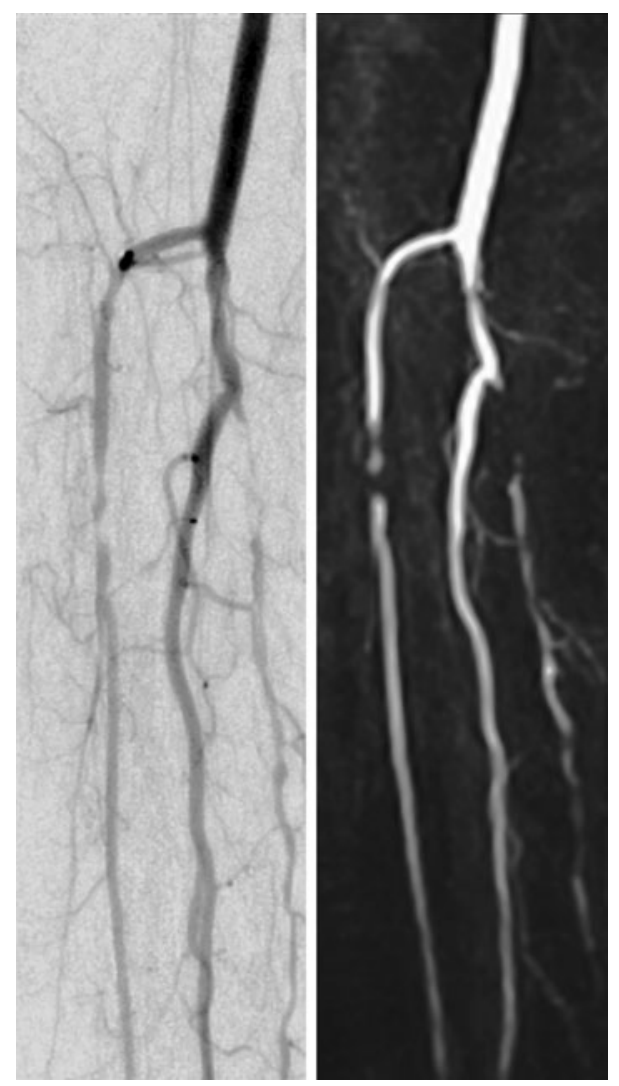

Fig. 2 Example of DSA versus NE-MRA. Patient is an 83-year-old man with Fontaine stage 2a prior to PTA: amplification of right calf trifurcation with severe stenosis and occlusion (Segmental subj. image quality reader $1 / 2: 2 / 2$ )

- Blinded stenosis grading:

For the assessment of stenosis, MIP images after the subtraction were used. Before the blinded stenosis grading, a preselection of lesions was necessary due to the presence of multiple stenosis in the patient collective and unpredictable image quality. Furthermore, the preselection was intended to reduce the bias between the comparison of intrainterventional and postinterventional image data.

Thus, in a pre-reading, the stenosis with the highest grade in each segment was selected by an independent pre-

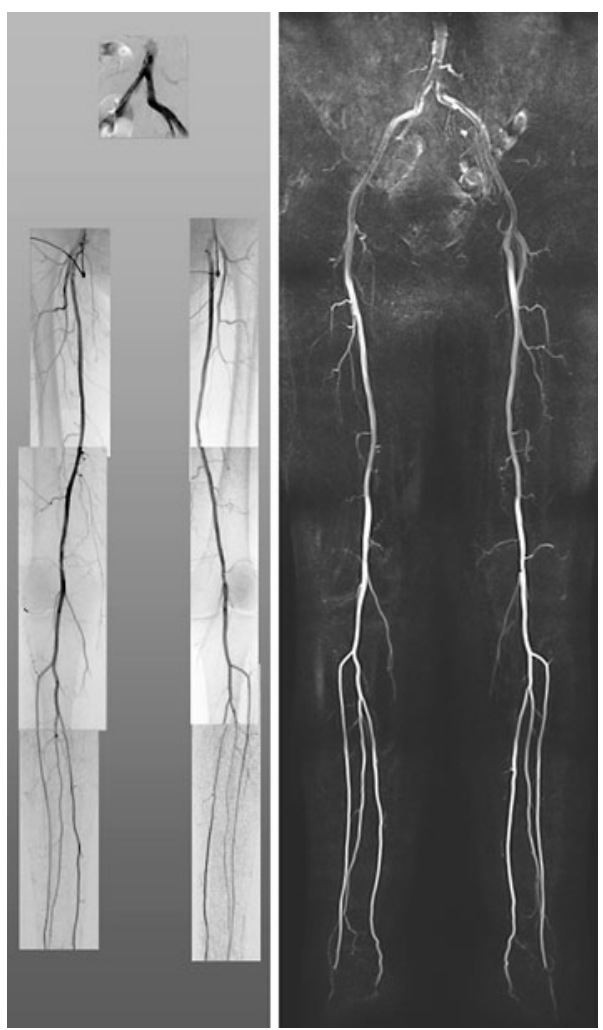

Fig. 3 Example of DSA versus NE-MRA. Patient is a 60-year-old woman with Fontaine stage $2 \mathrm{~b}$ : DSA-data for both legs available, comparison to NE-MRA MIP reconstructions (segmental subj. image quality for each step A B C D E and right/left side respectively: reader 1: $311111 / 31111$; reader 2: $322221 / 2222$ 1)

reader (with more than 10 years of MRA experience) resulting in a total of 66 stenoses being marked on the DSA and the corresponding NE-MRA image. The segmental distribution of pre-selected stenosis from $\mathrm{I}=$ common and external iliac artery to $\mathrm{V}=$ calf arteries was I: $3(5 \%)$, II: 12 (18 \%), III: 14 (21\%), IV: 10 (15\%), V: 27 (41\%) and included at least one stenosis per patient.

One blinded reader (interventionalist) rated each stenosis in the DSA images serving as a gold standard comparison. Two blinded radiologists independently determined the

Table 2 Acquisition parameters of all four NE-MRA steps

\begin{tabular}{|c|c|c|c|c|c|c|}
\hline \multirow{2}{*}{$\begin{array}{l}\text { Sequence } \\
\text { Step }\end{array}$} & \multicolumn{4}{|l|}{ NATIVE SPACE } & \multicolumn{2}{|c|}{ NATIVE TD scout } \\
\hline & Pelvis & Upper leg & Knee & Lower leg & Pelvis & Leg \\
\hline TR/TE (ms) & $300 / 38$ & $300 / 38$ & $300 / 36$ & $300 / 36$ & $29.7 / 3.3$ & $29.7 / 3.4$ \\
\hline FOV $\left(\mathrm{mm}^{2}\right)$ & $400 \times 380$ & $400 \times 362$ & $400 \times 325$ & $400 \times 310$ & $400 \times 262$ & $400 \times 212$ \\
\hline Slices & 64 & 72 & 72 & 72 & 1 & 1 \\
\hline Voxel size $\left(\mathrm{mm}^{3}\right)$ & $1.6 \times 1.3 \times 1.5$ & $1.6 \times 1.3 \times 1.3$ & $1.6 \times 1.3 \times 1.1$ & $1.6 \times 1.3 \times 1.1$ & $1.9 \times 1.6 \times 6.0$ & $1.9 \times 1.6 \times 6.0$ \\
\hline $\mathrm{BW}(\mathrm{Hz} / \mathrm{Px})$ & 868 & 868 & 868 & 868 & 260 & 260 \\
\hline TA (min:s) & $2: 03^{*}$ & $2: 19^{*}$ & $2: 16^{*}$ & $2: 21 *$ & $0: 21^{*}$ & $0: 28 *$ \\
\hline
\end{tabular}

* ECG-dependent, exemplary values of one subject 
presence and degree of each marked stenosis from NE-MRA images. Images were presented on film sheets and in pseudorandom order. A four-point grading-system was used:

- Grade 1: No stenosis or stenosis $<10 \%$ at marked position.

- Grade 2: $10 \%<$ Stenosis $<50 \%$.

- Grade 3: $50 \% \leq$ Stenosis $<90 \%$.

- Grade 4: Stenosis $\geq 90 \%$ or occlusion.

\section{Statistics}

Cohen's kappa was used to determine agreement between the two readers for evaluation of relative NE-MRA image quality and stenosis grading.

Comparison of NE-MRA image quality at the five evaluated arterial segments (I-V) was performed by using the unpaired nonparametric Wilcoxon rank-sum test. The Wilcoxon signed-rank test of matched pairs was applied to show the difference between stenoses grades determined by NE-MRA and the gold standard DSA for each reader. Statistical analysis was performed using Matlab (MathWorks, Natick, USA). For the evaluation of differences, statistical significance was established at $p<0.05$.

\section{Results}

The relative image quality of NE-MRA compared to DSA was rated $2.7 \pm 1.1$ by reader 1 and $3.0 \pm 1.0$ by reader 2 , respectively. Of the evaluated arterial segments, $40 \%$ and $44 \%$ showed good or sufficient image quality (grades 1 and 2), according to the readers' respective assessments. In $31 \%$ and $42 \%$, respectively, an inadequate segmental image quality was observed. The kappa-value for interobserver agreement was 0.34 (Table 3).

Comparing the image quality of different vessel segments (I-V), the grades for images of the iliac arteries-as graded by reader 1 -are lower than the quality grades of the other segments, which was statistically significant compared to the distal superficial femoral artery (III) and the calf arteries $(\mathrm{V})(p<0.05)$. No significant differences between the grades for arterial segments II-V were observed by reader 1 . The grades of reader 2 did not reveal any differences between the five arterial segments (Table 3). Example images of segment $\mathrm{V}$ with different quality grades are shown in Fig. 4. An overview of the results regarding image quality of NE-MRA is given in Table 3.

Blinded stenosis grading: The grades of the pre-marked stenosis were significantly higher in NE-MRA than in DSA images with mean grades of $3.0 \pm 0.7$ (reader 1 ,
Table 3 Segmental image quality reading

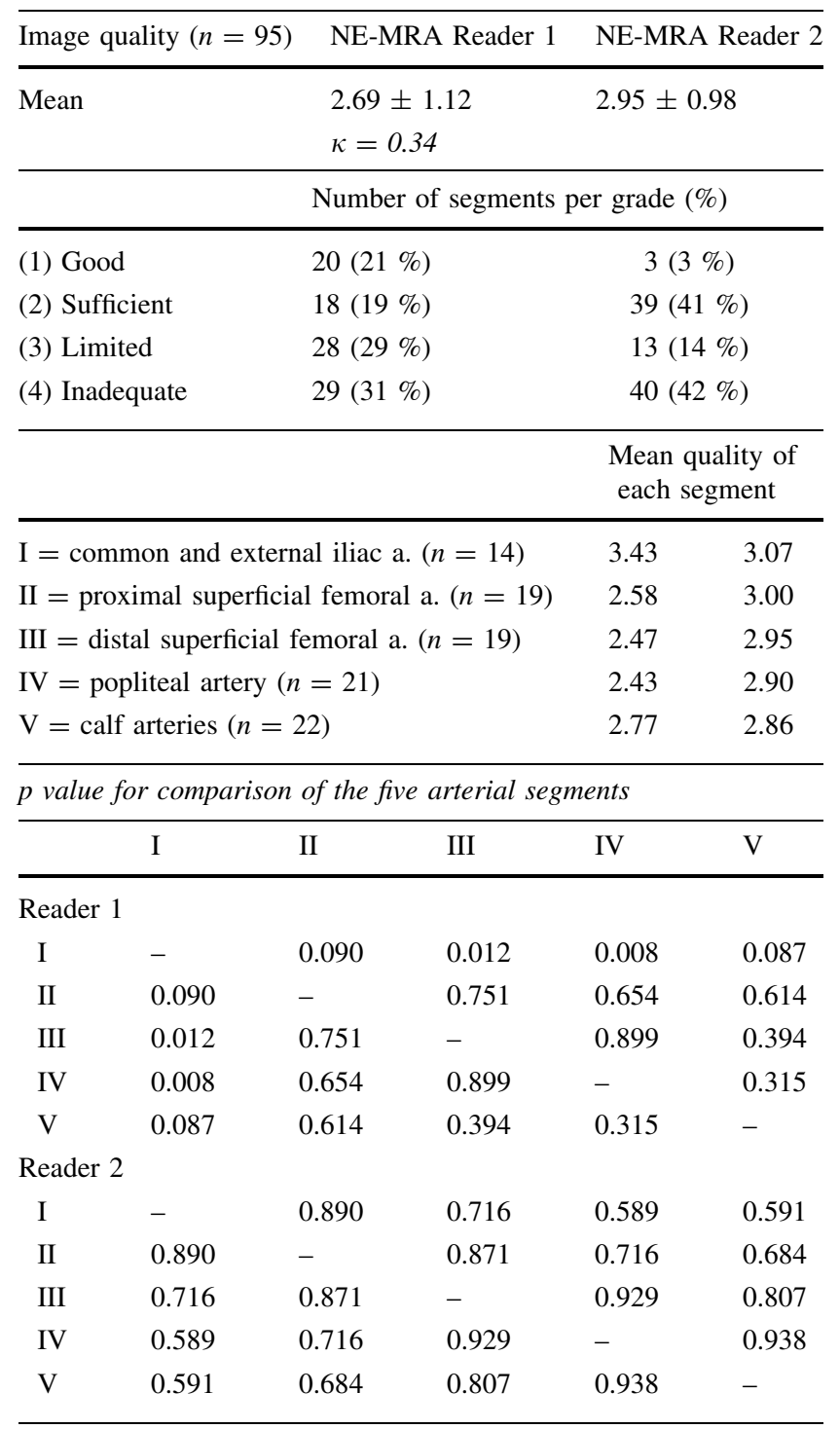

$p<0.001$ ) and $3.1+0.8$ (reader $2, p<0.001$ ) for NE-MRA compared to a mean grade of $2.7 \pm 0.8$ for DSA. There was a good agreement between the two readers regarding the stenosis rating $(\kappa=0.59)$.

The gold standard DSA revealed 10/66 (15\%) stenoses of grade 4, whereas with NE-MRA $16(24 \%)$ and 17 (26\%) segments were rated as grade 4, respectively. Relevant stenoses, which correspond to grades 3 and 4, were read in $53 \%$ of DSA images and 74 and $61 \%$ of NE-MRA image data, respectively (Table 4).

The number of stenoses rated as equal/higher/lower/notassessable in NE-MRA images in comparison to the gold standard DSA was for reader 1: 25 (38\%), $30(45 \%)$, $9(14 \%)$ and $2(3 \%)$; and for reader 2: 26 (39\%), 22 (33\%), $5(8 \%)$, and $13(20 \%)$. The 13 stenoses which were rated as not-assessable by reader 2 included the two rated as such by reader 1 . These two non-assessable 
Fig. 4 DSA versus NE-MRA from four different patients (Age/Gender/Fontaine stage prior to PTA a $60 / f / 2 b$, b $67 / \mathrm{m} / 2$ a, c $76 /$ f/inapplicablebypass stenosis of contralateral side, not shown d 56/f/2b).

Good (a) to inadequate

(d) image quality of calf arteries with grading of reader $1 /$ reader 2: a $1 / 1$, b $2 / 2$, c $3 / 3$, d $4 / 4$

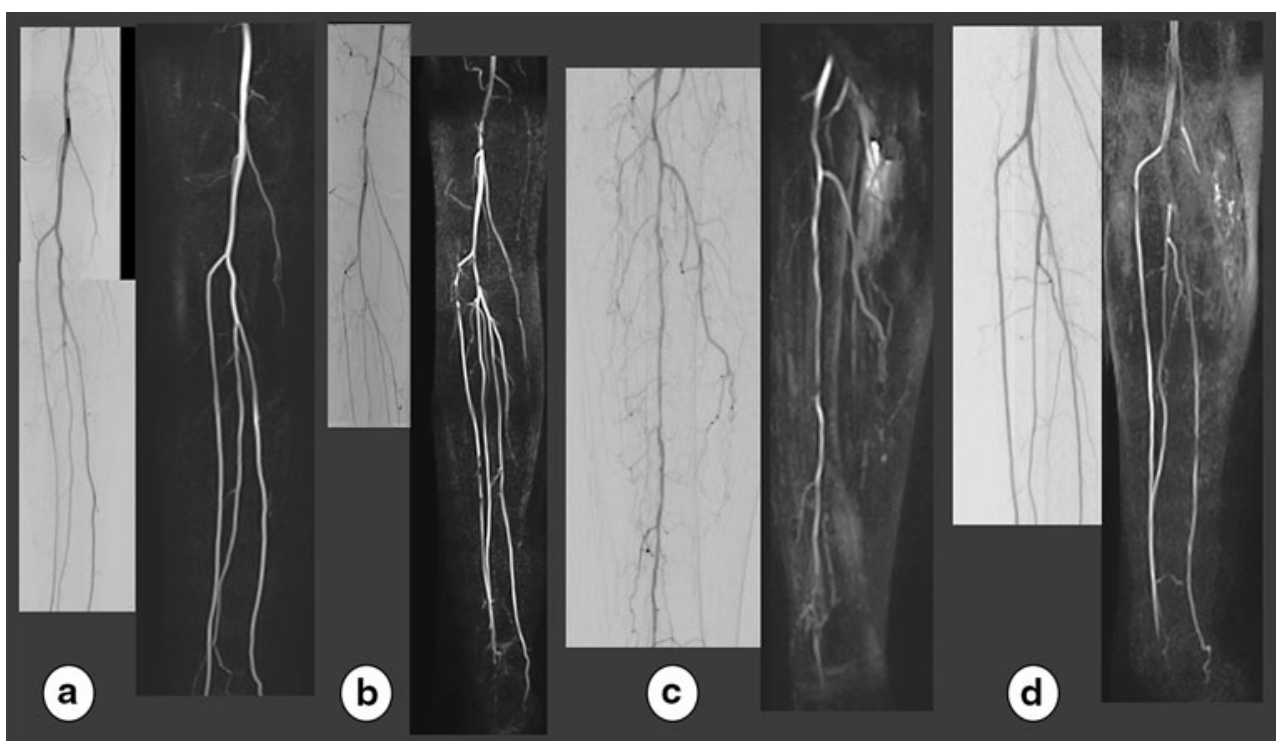

Table 4 Stenosis grading of preselected stenoses

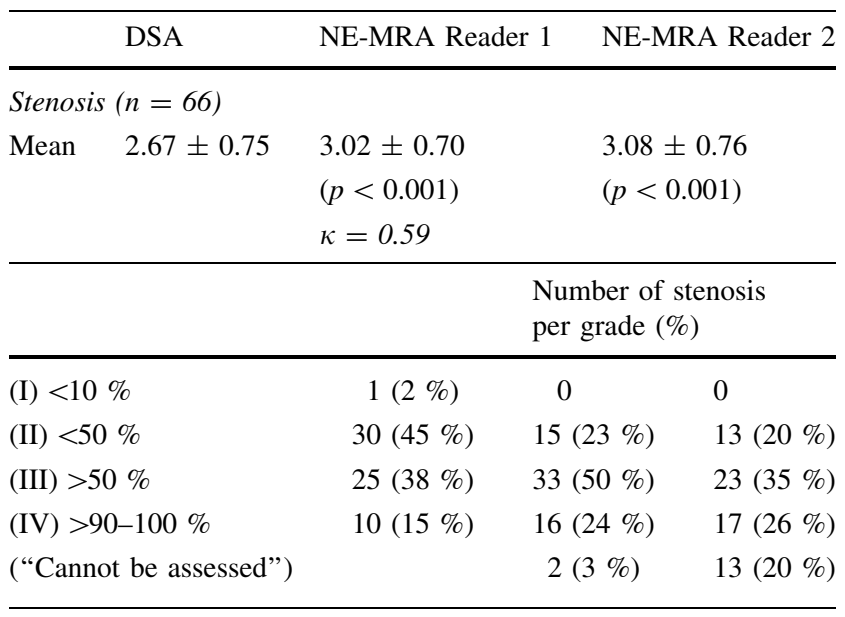

stenoses were rated as grade 2 and 4 in DSA. Eleven stenoses were only rated by reader 1 , and included lesions from grade 2-4 in DSA (mean 2.54). An overview of all results regarding the stenosis rating in NE-MRA and DSA is given in Table 4.

\section{Discussion}

Improvements in MR hardware and software, coupled with concerns about the safety of gadolinium-based contrast agents, have contributed to a renaissance of interest in nonenhanced MR angiography [16]. This study of the new technique, NATIVE SPACE, for patients with severe PAOD can be seen within this context.

The study revealed a limited number of arterial segments with good or sufficient visualization: in cumulative percentage, 40 and $44 \%$, respectively (reader 1,2 ). Of the remaining segments, 31 and $42 \%$, respectively, were graded as inadequate quality and another 30 and $14 \%$, respectively, were found to be of limited quality. Thus, less than half of all compared segments provided clinically reliable image data in this patient collective.

Several factors may have contributed to the high percentage of poor visualization. First, flow disturbances due to multiple stenoses may have hampered visualization quality of the flow-dependent technique in this collective. Second, motion artifacts and errors with ECG triggering may have contributed to the poor visualization. Finally, since only the subtracted MIPs were evaluated, visual information might have been removed by subtracting systolic from diastolic image data.

Iliacal segments showed slightly worse mean quality than peripheral segments. The reasons for this finding could be manifold. The influences of bowel motion, breathing and relatively reduced signal due to greater distance between the RF coil and the main vessel are likely to have interacted, especially in large patients.

In addition, we have not used velocity-dependent gradient flow compensation or spoilers in different stations, as indicated by Miyazaki et al. [17] and Nakamura et al [22]. This could have contributed to the lower quality in the proximal segments.

The stenosis reading revealed a tendency towards overestimation of stenosis. Whereas the gold standard DSA revealed $53 \%$ of preselected stenosis with grades of III $(>50 \%)$ and IV (>90\%), NE-MRA resulted in $74 \%$ and $61 \%$, respectively, from reader 1 and 2 . The effect is also known from other (CE-) MRA techniques, and is considerably present with this technique. The overestimation of stenosis in comparison to DSA was significant for both readers, however a few cases of underestimation were also 
present (reader 1/2: $14 \% / 8 \%$ ). Again, overestimation of stenosis may be reduced by reading the diastolic images, which show both bright arteries and veins [22].

Furthermore, the stenosis reading revealed a relatively high number of preselected stenoses which were graded as not assessable ( $3 \%$ reader 1 vs $20 \%$ reader 2 ). This may reflect the limited visualization quality with consecutive interpretation inconvenience.

Interobserver agreement was marginally good for stenosis estimation and moderate for segmental quality. We assume that the limited agreement may reflect the challenge of judging vessel segments with severe pathology accompanied by certain signal inhomogeneity.

In 2010, a study of the new NE-MRA technique in eight healthy volunteers and three patients was published [15]. It included a rough theoretic comparison of the new technique to newer HASTE-based ECG triggered approaches $[17,18]$ as well as to older NE-MRA techniques, such as TOF- and phase-contrast MRA, which need relatively long acquisition times and are limited in view of peripheral vessel imaging. NATIVE SPACE uses a variable flip angle and nonselective rectangular refocusing pulses, resulting in reduction of readout duration [23-25]. It subsequently decreases vessel blurring and performs more accurate data sampling [23, 24]. Lanzman et al. [15] concluded that the new NE-MRA technique was promising in demonstrating a high percentage of excellent and good quality segments, slightly depending on acquisition scheme (e.g. up to $100 \%$ of excellent subjective image quality of femoral artery NEMRA imaging). Another NE-MRA technique for peripheral arteries, quiescent-interval single-shot (QISS), has been proposed using 2-dimensional balanced steady-state free precession [26]. The main advantage of QISS is its short acquisition time. First studies suggested good agreement with CE-MRA [27].

Furthermore, several studies of peripheral NE-MRA with an ECG triggered approach comparable to the technique used in this study have been published.

Lim et al. [18] reported a study about NE-MRA at $1.5 \mathrm{~T}$ of the calf arteries of 36 patients in comparison to CEMRA and obtained accurate imaging of the calf arteries when technically successful. They reported a total of 17 patients $(47.2 \%)$ as having poor diagnostic confidence.

A study by Haneder et al. [19] evaluated NE-MRA of the calf station at $3 \mathrm{~T}$ in 36 patients in comparison to CE-MRA and came to the conclusion of superiority of CE-MRA, but high negative predictive value of NE-MRA, if technically successful. In that study, 122/288 (42\%) unassessable segments were reported.

Both studies used a different comparison standard (CE-MRA instead of DSA in our study) and slightly different definitions of grading category, besides differing patient collectives. However, the reported results correspond to our study results in terms of low segmental quality. Furthermore, a tendency towards overestimation of stenosis was reported similarly in those studies.

In further study comparing CE-MRA with NE-MRA of the lower extremities in PAOD patients and performing a modified turbo-spin-echo sequence, the authors found shorter acquisition times and significantly better image quality with CE-MRA. However, the sensitivity, specificity, and positive as well as negative predictive values were high and thus the author concluded that NE-MRA might be a valuable diagnostic alternative [20]. A recently published study assessed a similar NE-MRA technique (TRANCE) versus DSA in the lower extremities including the feet. The authors observed diagnostic capabilities of NE-MRA on the thigh and calf level whereas the diagnostic accuracy was insufficient in the pedal arteries [28].

The usage of a $3 \mathrm{~T}$ device by Haneder et al. [19] did not provide a relevant decrease of inadequate quality segments in comparison to the study by Lim et al. [18] and our study at $1.5 \mathrm{~T}$. This indicates that the relatively high number of inadequate visualization segments in NE-MRA may not be overcome by higher field strength alone.

Recently another study of the NE-MRA technique in comparison to CE-MRA at $1.5 \mathrm{~T}$ revealed high negative predictive value of $98 \%$ for detecting stenosis $>50 \%$ of leg and calf arteries in a collective of 50 patients with peripheral vascular disease [20], with $10 \%$ unassessable data sets, and positive predictive values of $67 \%$ for femoral and popliteal arteries and $69 \%$ of lower leg arteries, respectively.

In our study, only subtracted MIP images were evaluated. The same method was used by Lim et al [18]. A possible means of reducing false positive findings could be to evaluate diastolic images in cases of signal loss in the MIP images [22].

Despite relatively high numbers of insufficient quality segments in our study, we see some clinical potential of the technique:

- As a "fall back" after failed CE-MRA,

- In subjects with less flow disturbance (i.e. initial ruleout of stenosis), according to the reported high negative predictive value [18-20], and

- In second line, the technique may be useful for anatomic (pre-) imaging for future MRI-guided interventions.

Further research and advancement may widen the field of clinical relevance. We see the following aspects of future research interest in view of clinical usage of this NE-MRA technique:

1. Influence of flow conditions: A deeper understanding of the probably high influence of flow conditions on the NE-MRA outcome will help to improve the 
technique towards clinical demands. Specifically, questions on how far a reduced systolic flow velocity, a diminished systolic/diastolic flow difference or turbulent flow within diseased arteries influence the imaging outcome and how often those effects do appear in patients are of interest.

2. Prediction of NE-MRA success: A technique to figure out the probability of success of this NE-MRA algorithm is desirable in order to distinguish patients for which the NE-MRA approach is applicable from others. If the assumption of a primarily flow-dependent limitation of this technique's outcome can be proven, a prediction of imaging success by analysis of the cine scout or an initial (quick) arterial peak flow measurement at one or two heights might provide a decision as to whether an NE-MRA with reduced risk is applicable or not in a case by case manner.

3. Motion artifacts: Due to its underlying subtraction algorithm, the technique may benefit from additional motion correction steps to optimize subtracted data results. Alternatively, NE-MRA approaches that are not subtraction-dependent may decrease the number of insufficient quality outcomes. In the present study, we only evaluated the subtracted MIP images.

Additionally, further experience and technical improvements are likely to increase the number of qualitatively good or sufficient visualization segments.

Our study had limitations. A bias exists due to the fact that gold standard DSA was not available for all NE-MRA data, but is likely available for the more diseased side/ vessel parts of each patient. All NE-MRA were performed after interventional treatment. An inverse evaluation would have been more informative in terms of detection of clinically relevant stenosis. Even so, the inverse course of evaluation was abandoned. It would have led to a treatment delay and thus to an ethical and organizational problem.

Segments without stenosis were not included to avoid lesion mismatch between gold standard DSA and MRA reading within the limited number of preselected stenosis under multiple other stenoses. Accordingly, the data do not allow the assessment of false-positive stenosis.

Furthermore, we did not distinguish between a highgrade stenosis and an occlusion. It should be considered that it is, even for an experienced reader, difficult to impossible to differentiate a high-grade stenosis from an occlusion on DSA as gold standard. Furthermore, it is not required to determine this from a clinical perspective as no difference exists in therapy management. In both cases, the patient has to eventually undergo an invasive measure.

We prospectively abandoned additional quantitative analysis, because inhomogeneous flow conditions were expected. We did not expect reliable quantitative data of a flow dependent sequence within this limited collective. Additionally, the evidence of quantitative measurements in subtracted image data acquired with parallel imaging acceleration may have been questionable.

A major limitation is that NE-MRA techniques are intended to become an addition or alternative to CE-MRA. Thus, an additional comparison to CE-MRA would have been interesting. Even so, in this first pseudo-clinical setting we preferred the most reliable gold standard for comparison and abandoned an additional CE-MRA, as a further CE-MRA would have meant contrast material (including NSF-risk), considerable prolongation of scan time in patients with severe disease, or even prolonged hospitalization in the case of an additional scan.

\section{Conclusion}

The ECG triggered, non-enhanced peripheral MRA technique revealed unstable quality results in patients with severe PAOD, probably due to complex flow conditions with disturbing effects and motion artifacts. However, limited clinical potential can be assumed for certain indications in view of NSF risk reduction. Further advancement of contrast material-free MRA techniques remains clinically desirable, especially in view of stability against flow disturbance and motion artifacts.

Acknowledgments We thank Tanja Haas for her great support with MR scanning and image preparation. We also would like to thank Roger Yuh for proofreading the manuscript.

\section{References}

1. Prince MR, Narasimham DL, Stanley JC, Chenevert TL, Williams DM, Marx MV, Cho KJ (1995) Breath-hold gadolinium-enhanced MR angiography of the abdominal aorta and its major branches. Radiology 197:785-792

2. Goyen M, Herborn CU, Kröger K, Ruehm SG, Debatin JF (2006) Total-body 3D magnetic resonance angiography influences the management of patients with peripheral arterial occlusive disease. Eur Radiol 16:685-691

3. Bongartz G, Mayr M, Bilecen D (2008) Magnetic resonance angiography (MRA) in renally impaired patients: when and how. Eur J Radiol 66:213-219

4. Schoenberg SO, Knopp MV, Prince MR, Londy F, Knopp MA (1998) Arterial-phase three-dimensional gadolinium magnetic resonance angiography of the renal arteries. Strategies for timing and contrast media injection: original investigation. Invest Radiol 33:506-514

5. Wang Y, Lee HM, Avakian R, Winchester PA, Khilnani NM, Trost D (1998) Timing algorithm for bolus chase MR digital subtraction angiography. Magn Reson Med 39:691-696

6. Miyazaki M, Isoda H (2011) Non-contrast-enhanced MR angiography of the abdomen. Eur J Radiol 80:9-23

7. Bongartz G (2007) Imaging in the time of NFD/NSF: do we have to change our routines concerning renal insufficiency? MAGMA 20:57-62 
8. Lanzman RS, Voiculescu A, Walther C, Ringelstein A, Bi X, Schmitt P, Freitag S-M, Won S, Scherer A, Blondin D (2009) ECG-gated nonenhanced 3D steady-state free precession MR angiography in assessment of transplant renal arteries: comparison with DSA. Radiology 252:914-921

9. Grobner T (2006) Gadolinium-a specific trigger for the development of nephrogenic fibrosing dermopathy and nephrogenic systemic fibrosis? Nephrol Dial Transpl 21:1104-1108

10. Marckmann P, Skov L, Rossen K, Dupont A, Damholt MB, Heaf JG, Thomsen HS (2006) Nephrogenic systemic fibrosis: suspected causative role of gadodiamide used for contrast-enhanced magnetic resonance imaging. J Am Soc Nephrol 17:2359-2362

11. FDA NEWS RELEASE (2010) New warnings required on use of gadolinium-based contrast agents, enhanced screening recommended to detect kidney dysfunction. U.S. Food and Drug Administration http://www.fda.gov/NewsEvents/Newsroom/ PressAnnouncements/ucm225286.htm. Accessed 03 January 2012

12. Kos S, Huegli R, Hofmann E, Quick HH, Kuehl H, Aker S, Kaiser GM, Borm PJA, Jacob AL, Bilecen D (2009) MR-compatible polyetheretherketone-based guide wire assisting MR-guided stenting of iliac and supraaortic arteries in swine: feasibility study. Minim Invasive Ther Allied Technol 18:181-188

13. Hennig J, Weigel M, Scheffler K (2003) Multiecho sequences with variable refocusing flip angles: optimization of signal behavior using smooth transitions between pseudo steady states (TRAPS). Magn Reson Med 49:527-535

14. Busse RF, Hariharan H, Vu A, Brittain JH (2006) Fast spin echo sequences with very long echo trains: design of variable refocusing flip angle schedules and generation of clinical T2 contrast. Magn Reson Med 55:1030-1037

15. Lanzman RS, Blondin D, Schmitt P, Orzechowski D, Godehardt E, Scherer A, Mödder U, Kröpil P (2010) Non-enhanced 3D MR angiography of the lower extremity using ECG-gated TSE imaging with non-selective refocusing pulses-initial experience. Rofo 182:861-867

16. Miyazaki M, Lee VS (2008) Nonenhanced MR angiography. Radiology 248:20-43

17. Miyazaki M, Takai H, Sugiura S, Wada H, Kuwahara R, Urata J (2003) Peripheral MR angiography: separation of arteries from veins with flow-spoiled gradient pulses in electrocardiographytriggered three-dimensional half-Fourier fast spin-echo imaging. Radiology 227:890-896

18. Lim RP, Hecht EM, Xu J, Babb JS, Oesingmann N, Wong S, Muhs BE, Gagne P, Lee VS (2008) 3D nongadolinium-enhanced ECG-gated MRA of the distal lower extremities: preliminary clinical experience. J Magn Reson Imaging 28:181-189

19. Haneder S, Attenberger UI, Riffel P, Henzler T, Schoenberg SO, Michaely HJ (2011) Magnetic resonance angiography (MRA) of the calf station at $3.0 \mathrm{~T}$ : intraindividual comparison of non-enhanced ECG-gated flow-dependent MRA, continuous table movement MRA and time-resolved MRA. Eur Radiol 21:1452-1461

20. Mohrs OK, Petersen SE, Heidt MC, Schulze T, Schmitt P, Bergemann S, Kauczor H-U (2011) High-resolution 3D noncontrast-enhanced, ECG-gated, multi-step MR angiography of the lower extremities: comparison with contrast-enhanced MR angiography. Eur Radiol 21:434-442

21. Versluis B, Backes WH, van Eupen MGA, Jaspers K, Nelemans PJ, Rouwet EV, Teijink JAW, Mali WPTM, Schurink G-W, Wildberger JE, Leiner T (2011) Magnetic resonance imaging in peripheral arterial disease: reproducibility of the assessment of morphological and functional vascular status. Invest Radiol 46:11-24

22. Nakamura K, Miyazaki M, Kuroki K, Yamamoto A, Hiramine A, Admiraal-Behloul F (2011) Noncontrast-enhanced peripheral MRA: technical optimization of flow-spoiled fresh blood imaging for screening peripheral arterial diseases. Magn Reson Med 65:595-602

23. Xu J, Weale P, Gerhard L, Park J, Stoeckel B, Chen Q, Lim RP, Hardie A, Storey P, Hecht E, Mcgorty K, Lee VS (2008) A novel non-contrast MR angiography technique using triggered nonselective refocused SPACE for improved spatial resolution and speed. Proc Intl Soc Mag Reson Med 16:730

24. Lim RP, Storey P, Atanasova IP, Xu J, Hecht EM, Babb JS, Stoffel DR, Chang H, McGorty K, Chen Q, Rusinek H, Belmont HM, Lee VS (2009) Three-dimensional electrocardiographically gated variable flip angle FSE imaging for MR angiography of the hands at 3.0 T: initial experience. Radiology 252:874-881

25. Morita S, Ueno E, Masukawa A, Suzuki K, Machida H, Fujimura M, Kojima S, Hirata M, Ohnishi T, Kitajima K, Kaji Y (2009) Comparison of SPACE and 3D TSE MRCP at $1.5 \mathrm{~T}$ focusing on difference in echo spacing. Magn Reson Med Sci 8:101-105

26. Edelman RR, Sheehan JJ, Dunkle E, Schindler N, Carr J, Koktzoglou I (2010) Quiescent-interval single-shot unenhanced magnetic resonance angiography of peripheral vascular disease: technical considerations and clinical feasibility. Magn Reson Med 63:951-958

27. Hodnett PA, Koktzoglou I, Davarpanah AH, Scanlon TG, Collins JD, Sheehan JJ, Dunkle EE, Gupta N, Carr JC, Edelman RR (2011) Evaluation of peripheral arterial disease with nonenhanced quiescent-interval single-shot MR angiography. Radiology 260: 282-293

28. Gutzeit A, Sutter R, Froehlich JM, Roos JE, Sautter T, Schoch E, Giger B, Wyss M, Graf N, von Weymarn C, Jenelten R, Binkert CA, Hergan K (2011) ECG-triggered non-contrast-enhanced MR angiography (TRANCE) versus digital subtraction angiography (DSA) in patients with peripheral arterial occlusive disease of the lower extremities. Eur Radiol 21:1979-1987 